\title{
Quantum interference effects of out-of-plane confinement on two-dimensional electron systems in oxides
}

\author{
A. F. Santander-Syro $\odot,{ }^{1,}{ }^{*}$ J. Dai, ${ }^{1, \dagger}$ T. C. Rödel,,${ }^{1,2}$ E. Frantzeskakis, ${ }^{1}$ F. Fortuna $\odot,{ }^{1}$ R. Weht,${ }^{3,4,5}$ and M. J. Rozenberg ${ }^{6}$ \\ ${ }^{1}$ Université Paris-Saclay, CNRS, Institut des Sciences Moléculaires d'Orsay, 91405 Orsay, France \\ ${ }^{2}$ Synchrotron SOLEIL, L'Orme des Merisiers, Saint-Aubin-BP48, 91192 Gif-sur-Yvette, France \\ ${ }^{3}$ Departamento Física de la Materia Condensada, Comisión Nacional de Energía Atómica (CNEA), Avenida General Paz y Constituyentes, \\ 1650 San Martín, Argentina \\ ${ }^{4}$ Consejo Nacional de Investigaciones Científicas y Técnicas (CONICET), Buenos Aires, Argentina \\ ${ }^{5}$ Instituto de Tecnología Sabato, Universidad Nacional de San Martín - CNEA, 1650 San Martín, Argentina \\ ${ }^{6}$ Université Paris-Saclay, CNRS, Laboratoire de Physique des Solides, Orsay 91405, France
}

(Received 12 April 2020; revised 2 June 2020; accepted 26 June 2020; published 3 August 2020)

\begin{abstract}
It was recently discovered that a conductive, metallic state is formed on the surface of some insulating oxides. First observed on $\mathrm{SrTiO}_{3}(001)$, it was then found in other compounds as diverse as anatase $\mathrm{TiO}_{2}, \mathrm{KTaO}_{3}$, $\mathrm{BaTiO}_{3}, \mathrm{ZnO}$, and also on different surfaces of $\mathrm{SrTiO}_{3}$ (or other oxides) with different symmetries. The spatial extension of the wave function of this electronic state is of only a few atomic layers. Experiments indicate its existence is related to the presence of oxygen vacancies induced at or near the surface of the oxide. We present a simplified model aimed at describing the effect of its small spatial extension on measurements of its threedimensional (3D) electronic structure by angular resolved photoemission spectroscopy. For the sake of clarity, we base our discussion on a simple tight-binding scheme plus a confining potential that is assumed to be induced by the oxygen vacancies. Our model parameters are, nevertheless, obtained from density functional calculations. With this methodology, we can explain, from a very simple concept of selective interference, the "wobbling," i.e., the photoemission intensity modulation and/or apparent dispersion of the Fermi surface and spectra along the out-of-plane $\left(k_{z}\right)$ direction, and the "mixed 2D/3D" characteristics observed in some experiments. We conclude that the critical model parameters for such an effect are the relative strength of the electronic hopping of each band and the height/width aspect ratio of the surface confining potential. By considering recent photoemission measurements, in light of our findings, we can get relevant information on the electronic wave functions and the nature of the confining potential.
\end{abstract}

DOI: 10.1103/PhysRevB.102.075101

\section{MOTIVATION}

Transition-metal oxides are fascinating materials which, over the last few decades, have been the source of countless surprises, such as the discovery of high-temperature superconductivity in cuprates [1], colossal magnetoresistance in manganites [2], metal-insulator transitions in vanadates [3], and, more recently, the resistive switching behavior in various simple and complex oxides [4]. Understanding many of their properties is at the heart of some of the most interesting phenomena and biggest challenges in quantum materials science.

Nowadays, modern fabrication methods of thin films have allowed the realization of heterostructures of these oxides. These developments bring the promise of observing new functionalities which may open the way for novel electronic devices. That is the realm of the emergent field of oxide-based

\footnotetext{
*andres.santander-syro@u-psud.fr
}

†Present address: Institute of Physics and Lausanne Centre for Ultrafast Science (LACUS), École Polytechnique Fédérale de Lausanne, CH-1015 Lausanne, Switzerland. electronics [5,6], and one of the current focuses of major interest is in neuromorphic devices [7].

Among the recent highlights in the study of complex oxide heterostructures, a major breakthrough was the realization, in 2004, of a two-dimensional electron gas (2DEG) at the interface between two well-known, insulating, and nonmagnetic oxides, $\mathrm{SrTiO}_{3}$ and $\mathrm{LaAlO}_{3}$ [8]. This triggered a burst of research and new surprises; for instance, the remarkable finding of a similar 2DEG at the bare surface of $\mathrm{SrTiO}_{3}$ (STO) $[9,10]$. This discovery was rapidly followed by similar findings in a variety of other insulating materials, such as anatase [11,12], one of the stable phases of $\mathrm{TiO}_{2}$, ferroelectric $\mathrm{BaTiO}_{3}$ [13], orthorhombic $\mathrm{CaTiO}_{3}$ [14], and the strong spinorbit-coupled $\mathrm{KTaO}_{3}$ [15]. In STO, it was also found in other surfaces, particularly on the highly anisotropic (110) [16] and highly polar (111) [17] ones.

Since the initial reports, a great deal of progress has been made to investigate the origin of the intriguing metallic states appearing at the bare oxide surfaces, along with their main properties, and on how to improve their quality. Experimental evidence strongly suggests that they are created by the presence of oxygen vacancies $\left(V_{O}\right)$ at or near the surface $[9,10,12-21]$. Such vacancies seem to have a double key role: 
(1) they provide the doping electrons to make the 2DEG (i.e., up to two electrons are released by each missing $\mathrm{O}$ atom), and (2) since they are effectively charged +2 , they also produce the confining potential to the electron gas [9]. This basic scenario has been validated by electronic structure calculations [18] and confirmed experimentally in well-controlled synchrotron measurements $[10,19]$, where the systematic effect of UV light to create $V_{O}$ at the surfaces was monitored. Oxygen vacancies might also play a nontrivial role in the electronic and magnetic properties of such 2DEGs, depending on their distance to the surface and to the surrounding transition-metal atoms [22].

A recent development of great practical relevance that provides further support to the aforementioned mechanism was the discovery that a thin capping layer of just a few angstroms thickness of a reducing agent, such as $\mathrm{Al}$ or $\mathrm{Eu}$ atoms, deposited on top of STO produces a very high-quality 2 DEG $[13,19,20]$. In this case, the reducing agent is oxidized by capturing oxygen ions from the STO, leaving on its (buried) surface the vacancies that realize the metallic state, and simultaneously creating a protective capping layer. This procedure demonstrated its significance by forming an electron gas even in oxide materials where the oxygen vacancies by themselves are not stable or believed to be unable to dope the conduction band [23], such as the surface of $\mathrm{ZnO}$ [21], or by realizing a magnetically tunable capping layer, such as $\mathrm{EuO} / \mathrm{SrTiO}_{3}$ [20]. Moreover, this procedure allowed the measurement of a gate-tunable superconducting state on the 2DEG state at the STO surface [24].

Beyond all these advances, there are several key open questions about these 2DEGs. For instance, what are the microscopic requirements for an oxide to realize a $2 \mathrm{DEG}$ at its surface when doped with oxygen vacancies? Moreover, the actual distribution of those vacancies is currently unknown. A related significant conundrum is how to estimate the depth of the metallic state, i.e., how deep down from the surface it reaches into the material. In principle, an ideal two-dimensional state would not have any band dispersion perpendicular to the surface (henceforth called $z$ axis), and should display tubular Fermi surfaces along $k_{z}$. Estimates from density functional theory calculations indicate that the potential extends only for four or five unit cells beneath the surface [9].

In this sense, assessments of the 2DEG's width from photoemission experiments, which can probe the $z$-axis dispersion, still remain controversial. Some reports claim that the state is essentially 2D, with the gas being quantum confined within about $2 \mathrm{~nm}$ and exhibiting subbands $[9,10]$. However, other photoemission works report a significant $z$-axis dispersion, which was interpreted as an intriguing "mixed 2D-3D dimensionality" of the confined state [25].

The present work will attempt to clarify some of these issues. We shall show that photoemission data along the $z$ axis encodes valuable information, which may reveal the qualitative nature of the confining potential at the surface. The more detailed the photoemission data, the better estimate of the potential one may obtain. In addition, we shall describe how the qualitative features of the $z$-axis wobbling (the photoemission intensity modulation and/or apparent dispersion) can be understood as the effect of the quantum interference of the wave-function eigenstates (standing waves) along the confinement direction. We shall discuss this feature in terms of both the spatial extension of the wave function of the 2DEG and the shape of the confining potential. Our aim is thus understanding the general effects of quantum confinement on the photoemission data along the out-of-plane direction.

Recently, Moser and co-workers also considered the question of the description of the 2DEG along the confinement out-of-plane direction. They developed a formalism [26,27] based on the damped free-electron final-state approximation and the assumption of an exponential confining potential. Their approach allowed them to extract information on the parameters of such exponential confinement potential from comparison to angle-resolved photoemission spectroscopy (ARPES) experimental data. They focused their study on InN and anatase $\mathrm{TiO}_{2}$.

Here we shall investigate this problem under a different light, namely, we incorporate a more realistic multiband structure, including the orbital character and the different effective masses of the subbands, and consider two qualitatively different types of potential wells, one rectangular and the other wedgelike, to explore which type of confinement best represents the more common experimental situations. We shall first focus on the paradigmatic case of the $\mathrm{SrTiO}_{3}(001)$ surface. It will be modeled using a tight-binding Hamiltonian, with parameters taken from density functional theory (DFT) calculations. We shall show that while the system is actually quantum confined, and hence strictly nondispersive along $k_{z}$, its electronic structure may appear as having an energy dispersion along $k_{z}$. We shall see how this is an unequivocal consequence of quantum interference effects that are different for different subbands, and that also depend on the binding energy. Significantly, we argue that the study of these " $z$-axis dispersions" can provide a clue to the longstanding issue of the lateral spatial extension of the 2DEG state. We will then apply our model to anatase.

\section{MODEL HAMILTONIAN FOR SrTiO}

Our model for $\mathrm{SrTiO}_{3}$ (STO) is based on a tight-binding Hamiltonian, representing the $t_{2 g}$ orbitals, whose hopping parameters we obtained from a fit to a DFT band structure calculation-see Appendix A. In STO, the valence bands have mostly oxygen character and are completely full, while the Ti $d$ orbitals occupy the lower conduction bands and are nominally empty, except when the system is doped. In the cubic symmetry, such as an undistorted perovskite, Ti $d$ orbitals split due to the octahedral environment into $t_{2 g}$ and $e_{g}$ levels. The $t_{2 g}$ manifold is usually lower in energy and closer to the Fermi energy, being the first levels to be occupied with doping. Mattheiss had already shown how these states form three interpenetrating cigar-shaped Fermi surfaces, which grow with doping, along the main crystallographic axes of the cubic Brillouin zone [28]. The cigar shape emerges because one of the three $t_{2 g}$ states has a heavy mass (a small curvature in a $E$ vs $k$ plot) along each direction in reciprocal space, while the other two form light bands in the same direction. In a perfect cubic symmetry, the three $t_{2 g}$ states should be degenerate at $\Gamma$. However, the presence of small tetragonal distortions and, even more importantly, the 
spin-orbit interaction slightly breaks this degeneracy. Since these effects are relatively small in $\mathrm{SrTiO}_{3}$, and for the sake of keeping our model as conceptual as possible, we shall not consider these details for the moment.

We complement our STO 2DEG surface model by adding an attractive phenomenological potential, which is supposed to be experimentally originated by the oxygen vacancies. This potential only depends on the perpendicular-to-the-surface $z$ direction, $V\left(z_{i}\right)$, where the surface is assumed at $z_{0}=0$, and each subsurface lattice layer is at $z_{i}$. We then solve the lattice model and compute all the eigenvalues (energies) and eigenvectors (wave functions) of the confined states as a function of their in-plane momenta $k_{\|}$for each subsurface layer $z_{i}$, and obtain their $k_{z}$ dependence by Fourier transformation. Note that due to the loss of symmetry along $z, k_{z}$ is not a good quantum number for the wave functions of the 2DEG. Nevertheless, the lack of symmetry does not prevent the Fourier transformation along the perpendicular direction, which is merely a change of basis. Hence, we use it here to parametrize, as done in experiments, the measured photon-energy dependence (i.e., the out-of-plane electronic structure) of the photoemission intensity.

As mentioned before, we will consider here two prototypical forms for the potential, i.e., a rectangle and a wedge, with their width and depth taken as adjustable parameters. These shapes are schematic and qualitatively different. We may assume that the rectangular shape might be realized in a system where the oxygen vacancies are restricted throughout the first few layers below the surface/interface, as experimentally observed, e.g., at the interface $\mathrm{AlO}_{x} / \mathrm{TiO}_{2}$ (see the supporting information of Ref. [13]). On the other hand, we may consider the wedge shape as a simplified representation of an attractive potential that might emerge from a self-consistent Poisson-Schrödinger calculation [29]. As mentioned before, the issue of the actual distribution of oxygen vacancies at, or just beneath, the surface is still an open problem.

We also consider the related case of anatase $\mathrm{TiO}_{2}$, which has a different crystal symmetry. The same procedure described before to obtain the tight-binding Hamiltonian remains appropriate [30] — see Appendix B for details on the relevant DFT calculations.

\section{RESULTS AND ANALYSIS}

In the framework of a one-electron description of the electronic structure of the solid, using Fermi's golden rule and the dipole approximation, the photocurrent $I$ of electrons emitted with kinetic energy $E_{\text {kin }}$ from an initial state $\left|\psi_{i}\right\rangle$ of the 2DEG, after excitation with light of energy $\hbar \omega$ and polarization vector $\vec{\epsilon}$, can be expressed as [31]

$$
I \propto\left|\left\langle\psi_{f}|\vec{\epsilon} \cdot \vec{\nabla}| \psi_{i}\right\rangle\right|^{2} \times \delta\left(\varepsilon_{i}+\Phi+E_{\mathrm{kin}}-\hbar \omega\right),
$$

where $\left|\psi_{f}\right\rangle$ is the final state of the optical transition and $\Phi$ is the work function.

Following previous works [26,27,32-35], we approximate the final state as a plane wave in vacuum, with momentum $\vec{k}_{f}$ and damping over a length $\lambda$ from the surface $(z=0)$ towards the bulk, to account for the finite escape length of the photoelectrons,

$$
\psi_{f}(\vec{r})=e^{\left(i \vec{k}_{f}-\hat{u}_{z} / \lambda\right) \cdot \vec{r}}=e^{i \vec{k}_{f} \cdot \vec{r}} e^{-z / \lambda},
$$

where $\hat{u}_{z}$ is the unit vector along the surface normal.

As the operator $\vec{\nabla}$ is Hermitian, we can let it act on the damped free-electron final state of the matrix element, given by Eq. (1), giving

$$
\left\langle\psi_{f}|\vec{\epsilon} \cdot \vec{\nabla}| \psi_{i}\right\rangle=\vec{\epsilon} \cdot\left(i \vec{k}_{f}-\frac{\hat{u}_{z}}{\lambda}\right)\left\langle e^{\left(i \vec{k}_{f}-\hat{u}_{z} / \lambda\right) \cdot \vec{r}} \mid \psi_{i}\right\rangle
$$

The geometric prefactor in this expression depends only on the angle between the light polarization $\vec{\epsilon}$ and the direction $\vec{k}_{f}$ of the emitted electron. If the energy of the initial state is kept constant, as done experimentally when mapping the out-of-plane Fermi surfaces, increasing the photon energy to probe the $k_{z}$ dependence of the electronic structure will result in a larger final-state kinetic energy and, consequently, a larger final-state momentum $\vec{k}_{f}$. Hence, far from any resonances, the main effect of increasing the photon energy will simply be to smoothly change the amplitude of the geometric prefactor and to increase the size of reciprocal space probed by photoemission. Thus, we can approximate the photocurrent as $[26,27,34,35]$

$$
I\left(\vec{k}_{f}\right) \propto\left|\int_{0}^{\infty} e^{i \vec{k}_{f} \cdot \vec{r}} e^{-z / \lambda} \psi_{i}(\vec{r}) d \vec{r}\right|^{2} .
$$

In other words, within the free-electron final-state approximation, the photocurrent is proportional to the amplitude squared of the Fourier transform of the damped initial-state wave function. The inclusion of a finite damping length complicates the analysis a bit without altering the qualitative results [27] — see the Appendix, Fig. 7. As we wish to focus on the essential consequences of quantum interferences induced by confinement on the electronic structure along $k_{z}$, we will henceforth neglect the effects of finite photoelectron escape length and dipole matrix elements. In this way, the importance of the more superficial states will be immediately evident. Thus, in what follows, we will simply model the photoemission intensity as

$$
I(\vec{k}) \propto\left|\tilde{\psi}_{i}(\vec{k})\right|^{2},
$$

i.e., as the modulus squared of the initial state's Fourier transform $\tilde{\psi}_{i}(\vec{k})$ [33-35].

In Figs. 1 and 2, we show our modeling results for $\mathrm{SrTiO}_{3}(001)$ using both a rectangular and a wedge confining potential, respectively. In the corresponding Figs. 1(a) and 2(a), we show the band structures of our system along the $k_{x} / k_{y}$ coordinates-the $x$ and $y$ directions are degenerate by symmetry, and we will note them as $k_{\|}$when the states are degenerated (or almost degenerated) in the whole plane. The multiple bands above the zero-energy level (taken here as the lowest conduction state in the bulk) are bulk states. Their multiplicity comes from the real-space representation of our slab along $z$, which will become a shaded region in a true semi-infinite system. Bands below zero energy are the subbands, pulled down by the confining potential. The number of subbands and their energy positions will depend on the shape and depth of the confining potential, while their curvatures (i.e., effective masses) are essentially given 

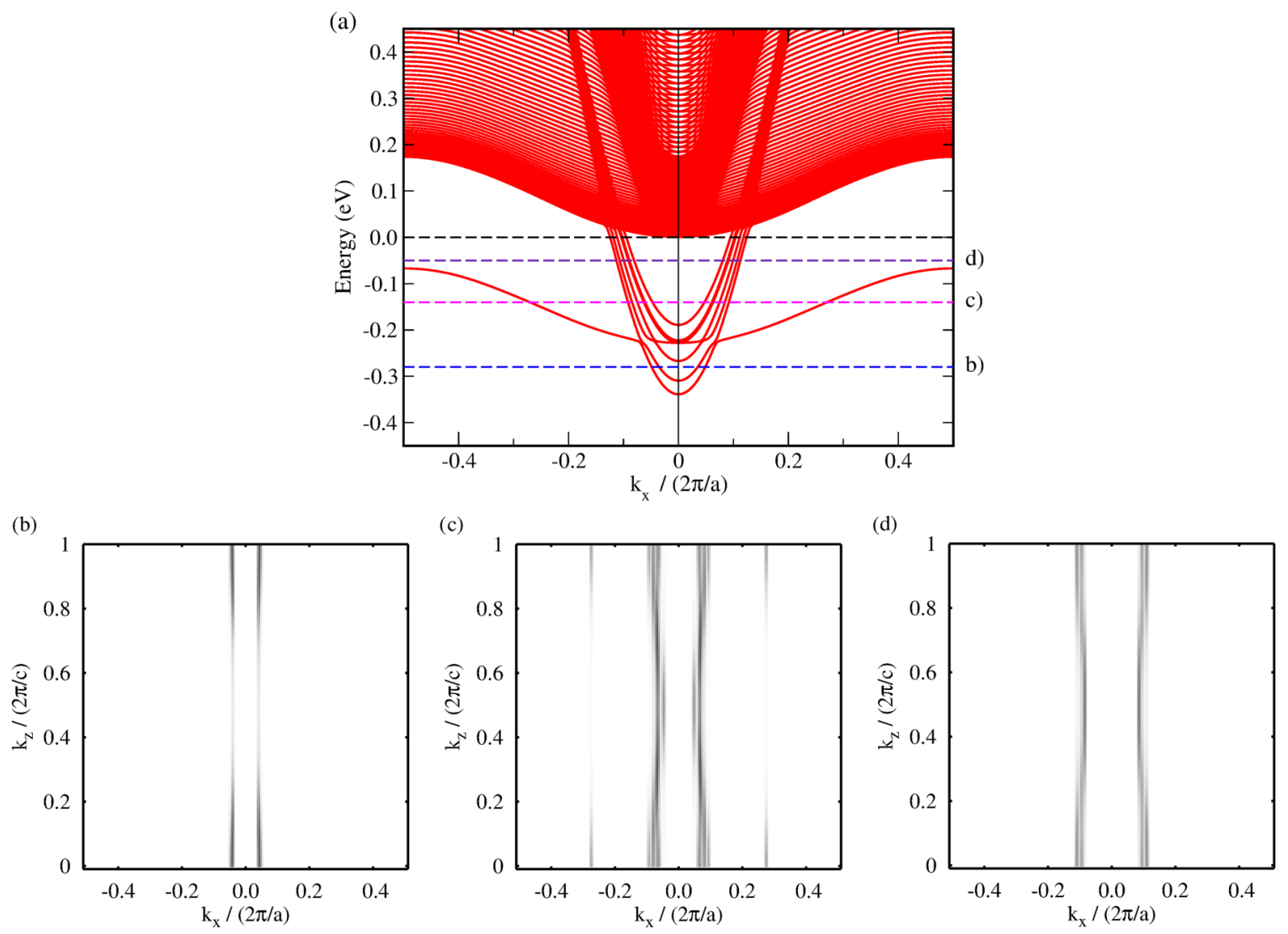

FIG. 1. Band structure and Fermi surfaces (amplitude squared of the electron wave functions) for a $\mathrm{SrTiO}_{3}$ slab taking a rectangular confinement potential of depth $0.35 \mathrm{eV}$ and a five-unit-cell width. (a) Band structure along a $k_{x}$ path. Different energy levels indicate different amounts of doping (oxygen vacancies). Explicit values here are (b) $-0.28 \mathrm{eV}$, (c) $-0.14 \mathrm{eV}$, and (d) $-0.05 \mathrm{eV}$. Zero of the energy axis is fixed to the lower conduction state for the bulk. (b)-(d) The corresponding Fermi surfaces in the $k_{x} / k_{z}$ plane.

by the respective bulk bands from which they come from. In both the rectangular and wedgelike potential cases, we chose the parameters of the wells to pull down a similar number of bands, i.e., five light and one heavy. The relative asymmetry in the number of bands pulled down can be most easily understood in terms of the perpendicular effective mass, defined as proportional to the inverse of the hopping integral along the out-of-plane direction. Thus, bands having a heavy mass along $k_{z}$, corresponding by $t_{2 g}$ symmetry to light bands along $k_{x}$ and/or $k_{y}$, are relatively more easily pulled down [9].

In Figs. 1(b)-1(d) and Figs. 2(b)-2(d), we plotted the corresponding Fermi surfaces (visualized as the amplitude squared of the electron wave functions), on the $k_{x} / k_{z}$ plane, for some given energy levels indicated by the dashed horizontal lines in Figs. 1(a) and 2(a). These energy levels would correspond to some Fermi energies given by the occupation of the subbands. In an actual experiment, this level will depend on total doping induced by the dosing of oxygen vacancies, or eventually by an applied gate potential.

We first focus on Fig. 1. At first glance, some of the Fermi-surface sheets appear as weakly dispersive in $k_{z}$, corresponding to quasi-2D tubular Fermi surfaces. However, upon closer inspection, we can notice quite interesting effects. In fact, while each of the Fermi sheets is strictly nondispersive, the amplitude squared of the electron wave functions show an intensity modulation in $k_{z}$, due to interference effects of each wave function in the confining potential well, that gives the impression of an incipient Fermi-surface dispersion, most visible in Figs. 1(c) and 1(d). In contrast, such an effect is less apparent in Fig. 1(b). The reason can be traced back to the number of bands that are crossing the Fermi energy, their separation, and orbital characters. We see that there are only two bands crossing $E_{F}$ in Fig. 1(b), while a bundle of at least five bands crosses it in Figs. 1(c) and 1(d). This is an interesting observation since the resolution in ARPES experiments of all possible subbands predicted by calculations remains an experimental challenge [29]. Thus, as shown by our simple modeling, even a seemingly small Fermi-surface dispersion along $k_{z}$ in the experimental data would be actually due to the combined effects, on multiple subbands, of intensity modulations of their electron wave functions and experimental resolution broadening. Hence, a detailed analysis of the outof-plane experimental data along the lines exposed here would provide a means to probe, or even unveil, different subbands beyond the nominal experimental resolution.

Another interesting feature is that the out-of-plane intensity modulation of a given state depends on its "effective mass" (inverse of the hopping integral) along $k_{z}$. For instance, the modulation of the outmost Fermi surface in Fig. 1(c) is stronger than the one in Fig. 1(b). In fact, as seen in Fig. 1(a), while the former derives from a $d_{y z}$ state of heavy mass in $k_{x} / k_{y}$ but light mass in $k_{z}$, the Fermi surface in Fig. 1(b) stems 

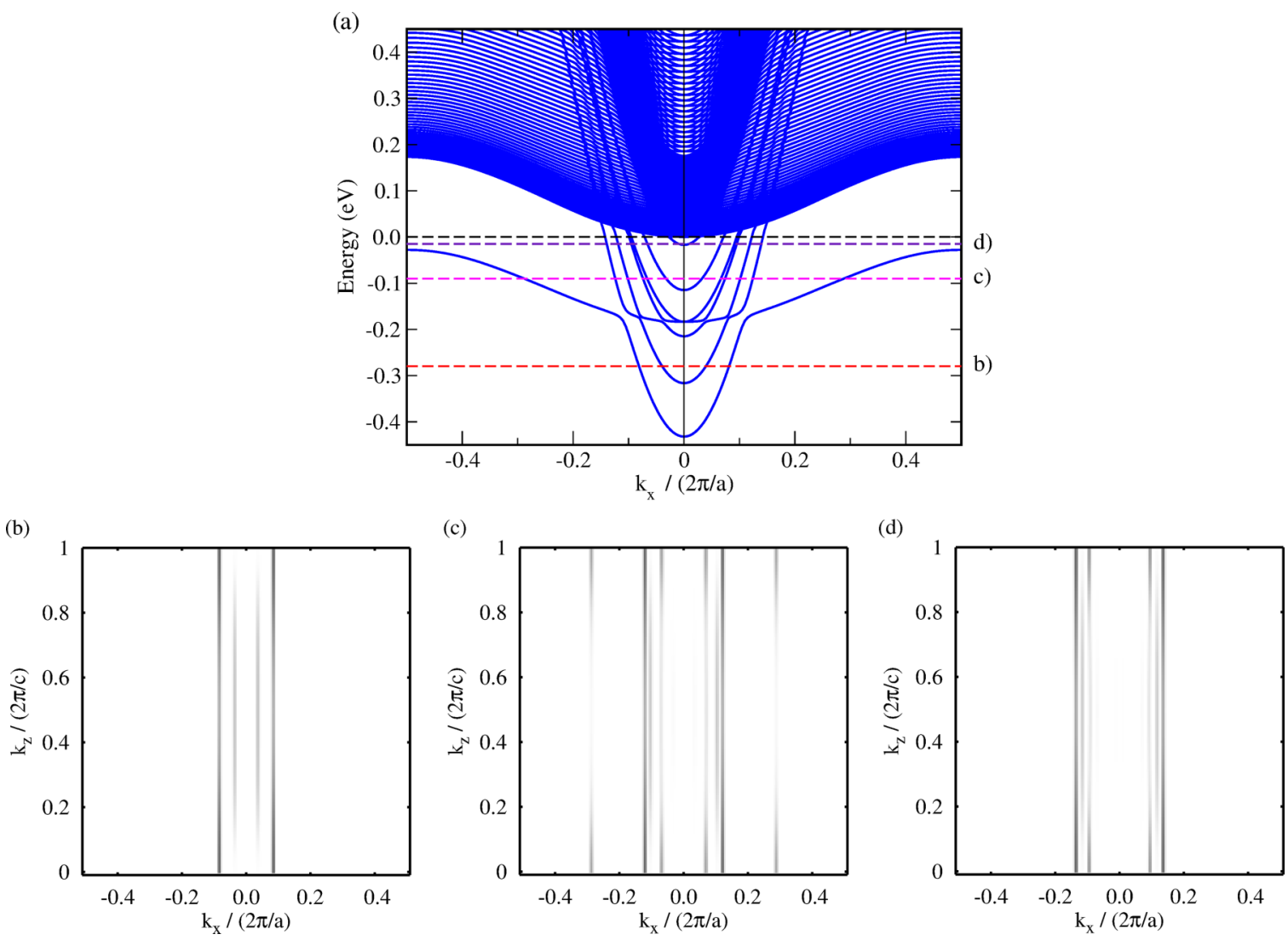

FIG. 2. Same as Fig. 1, but taking a wedge potential of depth $0.50 \mathrm{eV}$ and, again, a five-unit-cell width. Explicit cutting energy levels are (b) $-0.28 \mathrm{eV}$, (c) $-0.09 \mathrm{eV}$, and (d) $-0.016 \mathrm{eV}$.

from the first couple of $d_{x y}$ subbands, which are light in $k_{\|}$but heavy in $k_{z}$. Thus, a light out-of-plane mass implies a stronger quantum modulation of its Fermi-surface intensity.

It is noteworthy that the results of Fig. 1(c) qualitatively resemble the data reported by Plumb et al. [25] for the out-ofplane Fermi surface of the 2DEG at the $\mathrm{SrTiO}_{3}(001)$ surface. Thus our analysis puts under a clearer light the puzzling interpretation of "mixed 2D-3D dimensionality" advanced by those authors, replacing it into the more precise context of a collection of tubular Fermi surfaces with different intensity modulations along $k_{z}$, all of which create the impression of a 3D Fermi surface dispersing in the out-of-plane direction for the in-plane heavy subband.

To gain further insight, we now turn to the wedge potential case that is shown in Fig. 2. The main qualitative observation upon comparison to the previous case is that the intensity modulation is also very strong; however, the wobbling dispersive effect along $k_{z}$ is now significantly less pronounced. The reason for the less pronounced wobbling can be traced to the fact that unlike the case of the rectangular potential, now the light subbands appear more separated from each other, as can be seen in Fig. 2(a). This is due to the nature of the wedge potential, which has the form $V\left(z_{i}\right)$, and therefore acts as a different crystal field for each subsurface lattice layer $z_{i}$. This "layer-dependent crystal field" fully breaks the quasidegeneracy of the rectangular potential case, where all sublayers are subject to the same potential $V_{o}$ up to the well thickness.
This is a very interesting and rather surprising observation since one may naively expect that a self-consistent PoissonSchrödinger treatment of the surface would be more appropriate [29]. However, in most data available for the $k_{z}$ dispersion of 2DEGs in oxides, one does observe a rather smooth but clear wobbling of the dispersion. We can illustrate this with previously unpublished ARPES data in $\mathrm{SrTiO}_{3}$, shown in Fig. 3. The excellent quality of the data is due to the surfacepreparation method, which consisted in capping the bare STO surface with an atomically thin layer of pure $\mathrm{Eu}[13,20]$. Here, we observe a clear modulation effect, especially for the outermost Fermi surface (corresponding to the in-plane heavy subband), in good qualitative agreement with the results of Fig. 1(c). So, a qualitative comparison with the simulations insinuates that the experimentally observed wobbling might be related to a more rectangularlike attractive potential acting on the first few subsurface layers, suggesting that the oxygen vacancies are distributed within those layers. However, we also note that other details seem to be better captured by the wedge potential. For instance, in Fig. 2(b), we see that the quantum interfere modulation picks up a stronger intensity near the $\Gamma$ point $\left(k_{z}=0\right)$, similarly as in the experimental data.

In this sense, note that previous experimental studies of $\mathrm{TiO}_{2}$ anatase showed that the oxygen vacancies responsible for the formation of the 2DEG and the confining potential well are approximately homogeneously distributed over around $1 \mathrm{~nm}$ below the surface (see Ref. [13], in particular, 

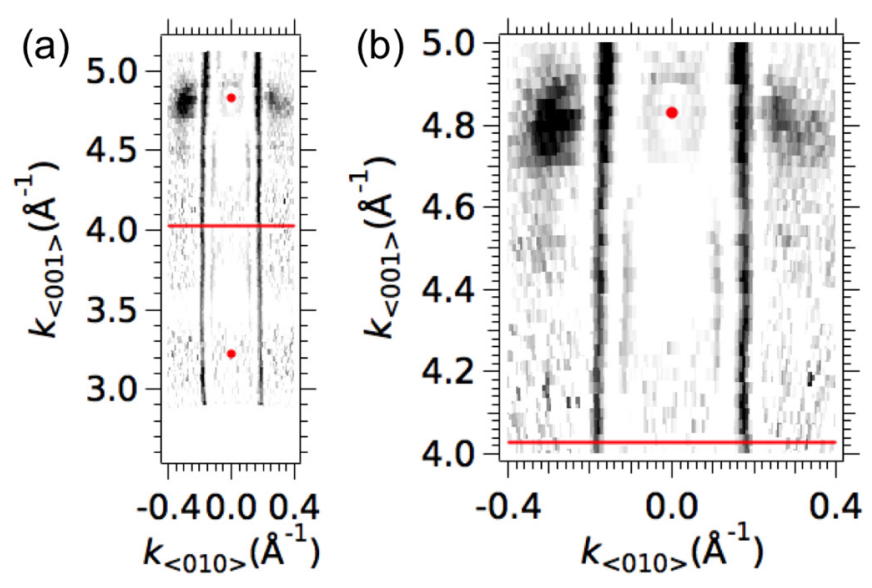

FIG. 3. (a) ARPES Fermi surface of $\mathrm{EuO}(1 \mathrm{ML}) / \mathrm{SrTiO}_{3}$ along the $k_{z}$ axis, acquired by varying the photon energy between 30 and $97 \mathrm{eV}$ in steps of $1 \mathrm{eV}$ using linear vertical photon polarization. An inner potential of $16 \mathrm{eV}$ was used in the calculation of the out-ofplane momentum [20]. The red discs and lines mark, respectively, the bulk $\Gamma$ points and the border of the bulk Brillouin zone. (b) Zoom over the upper part of the Fermi surface in (a). All data in this figure were acquired at $15 \mathrm{~K}$, and correspond (for better signal-tobackground ratio) to the negative part of the second derivative of the raw data.

Supplementary Fig. 5 and the related discussion). Thus, these vacancies would form a positively charged slab of finite thickness, comparable to the spatial extension of the 2DEG's electronic wave function, rather than a strictly $2 \mathrm{D}$ charged plane at the surface. While the latter would produce a wedgelike confining potential, the former would actually produce a quadratic (parabolic) confining potential in the region where the vacancies are distributed, followed by a linear wedgelike potential beneath such a region towards the bulk, and bound by an infinite potential barrier at the surface. Thus, in the region where the 2DEG states are located, the potential well due to vacancies would be more "rectangular," while it might become "wedged" beneath.

We leave the precise quantitative evaluation of the confining well that can best describe the experimental data for future research. Here, our goal is to emphasize the qualitative impli- cations of quantum mechanical confinement, in the simplest possible context, for the understanding of the out-of-plane ARPES data of 2DEGs in oxides.

To conclude our study, we shall consider the case of a different oxide, $\mathrm{TiO}_{2}$ anatase (001), which in contrast to STO has a tetragonal crystal symmetry. We also take this case to explore the effect of the depth of the well.

The conduction bands of bulk anatase $\mathrm{TiO}_{2}$ also have $t_{2 g}$-type character, like in STO. However, in contrast to the previous compound, the tetragonal symmetry breaks down their degeneracy and the orbitals separate in two groups, with one and two bands per atom in the unit cell. Moreover, also here the lower conduction band in the bulk has the complementarity of being light in the $k_{x} / k_{y}$ plane, but heavier along $k_{z}$-see Fig. 6.

In Fig. 4, we show the calculated band structures for two rectangular confining potentials, both of depth $0.35 \mathrm{eV}$ but with different widths along the $z$ axis. Figure 4(a) shows the case of five $\mathrm{TiO}_{2}$ layers, while Fig. 4(b) considers the case of $10 \mathrm{TiO}_{2}$ layers. Figure 4(c) shows the results for the $k_{z}$ axis dispersion for the thicker well. We observe that all the previously mentioned features arise, namely, a rather smooth wobbling of the dispersion for an energy cut that goes through a tightly packed bundle of subbands. Such a bundle would unlikely be experimentally resolved along the $k_{\|}$direction, but we see here that the $k_{z}$ data can provide hints to its existence.

\section{CONCLUSIONS}

We have considered a simple tight-binding Hamiltonian, with realistic hopping parameters derived from DFT, to build a model of a quasi semi-infinite lattice with a confining potential at the surface. We analyzed the qualitative differences for different shapes of the surface potential well, relevant for ARPES experiments of 2DEGs at the surface of transitionmetal oxides. Our analysis allowed us to provide a qualitative understanding of the interference phenomena along the $z$-axis direction originated in the few-layer quantum confinement of the 2DEGs.

Our results provided clarification of a puzzling "mixed dimensionality" state reported in $\mathrm{SrTiO}_{3}$ experiments. By the comparison of different confining potential shapes, we (a)

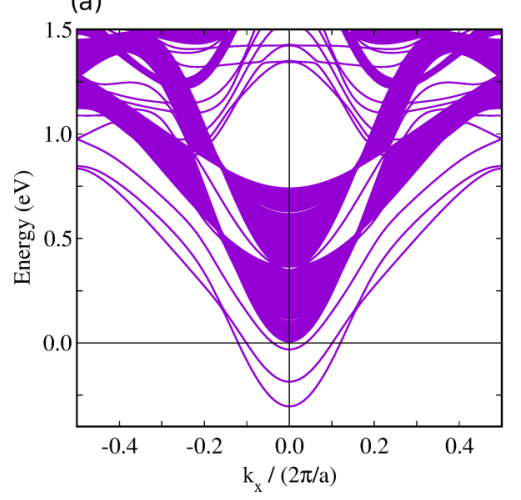

(b)

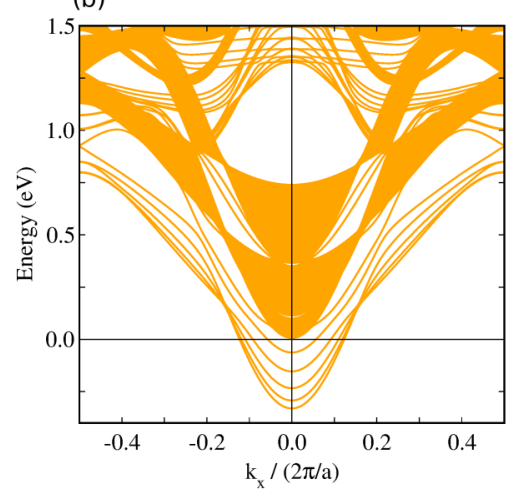

(c)

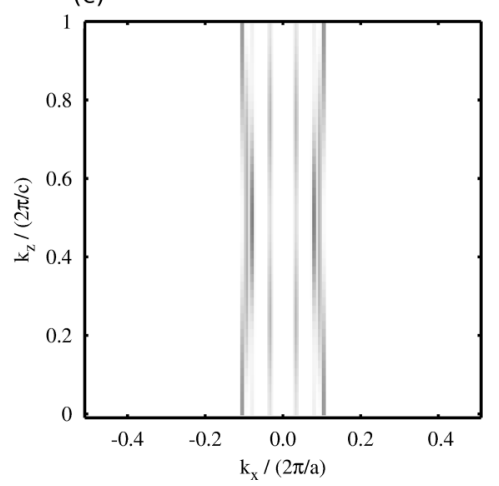

FIG. 4. Band structures and Fermi surface (amplitude squared of the electron wave functions) for an anatase $\mathrm{TiO}_{2}$ slab with a rectangular confining potential of depth $0.35 \mathrm{eV}$ and two different widths: (a) $5 \mathrm{TiO}_{2}$ layers and (b) $10 \mathrm{TiO}_{2}$ layers. (c) Corresponding Fermi surface for the case with 10 layers and $E_{F}=-0.10 \mathrm{eV}$. 
(a)

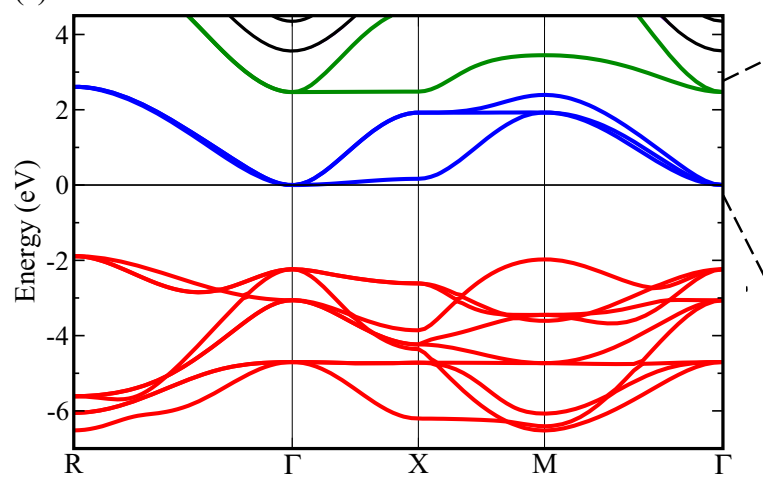

(b)

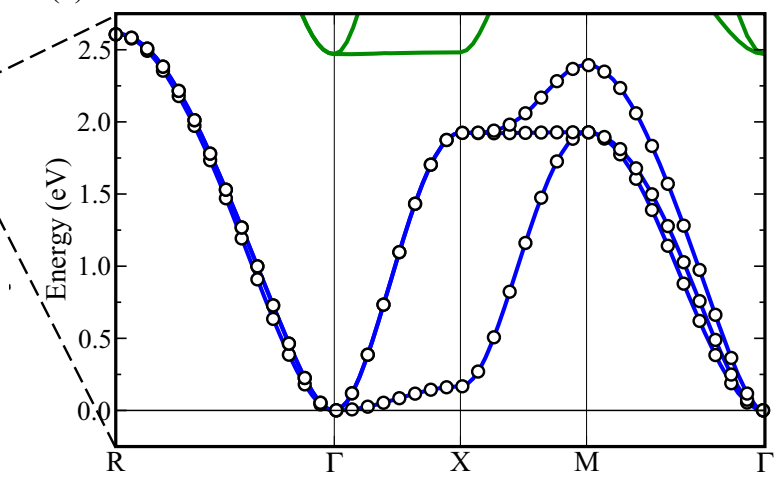

FIG. 5. (a) Standard DFT bands for $\mathrm{SrTiO}_{3}$. The calculation method is DFT, using the code WIEN2K. Red bands below the Fermi level correspond to states with mostly oxygen character. Blue bands between the Fermi energy and $\approx 2.5 \mathrm{eV}$, and green bands above $2.5 \mathrm{eV}$, correspond to the $t_{2 g}$ and $e_{g}$ manifolds, respectively. (b) Wannier fitting to the $t_{2 g}$ bands (black circles).

argue that, contrary to naive expectations, the rectangular well seems to better represent the more common experimental situation. This underlines the need to achieve a better understanding of the (self-)organization of the oxygen vacancies that originate the 2DEGs. We also discussed how the observation of a smooth wobbling of the bands along the $z$ axis, which so far has been minimally studied, may provide a valuable indication of the presence of subbands that are tightly packed, beyond the experimental resolution limit. In this spirit, we may envision that a careful fit of the ARPES data along the $z$ axis may provide detailed information on the shape of the confining potential and, henceforth, on the reorganization of the oxygen vacancies beneath the surface. This appears as an exciting future challenge within our reach.

\section{ACKNOWLEDGMENTS}

This work was supported by public grants from the French National Research Agency (ANR), Project LACUNES No. ANR-13-BS04-0006-01, the "Laboratoire d'Excellence Physique Atomes Lumière Matière" (LabEx PALM projects ELECTROX and 2DEG2USE) overseen by the ANR as part of the "Investissements d'Avenir" program (Reference No. ANR-10-LABX-0039), and the CNRS-CONICET 2015-2016 collaborative project AMODOX (Project No. 254274). R.W. gratefully acknowledges CONICET and ANPCyT (Grant No. PICT-2016-0402), Argentina, for partial support and Dario Carballido (FRED-CNEA) for computing assistance.

\section{APPENDIX A: DFT AND TIGHT-BINDING MODEL ${\text { FOR } \mathrm{SrTiO}_{3}}$}

DFT calculations of the $\mathrm{SrTiO}_{3}$ band structure were done starting from a standard all-electron density functional calculation, using, in our case, the WIEN2K code [36]. The obtained bands are shown in Fig. 5(a). We then projected the $t_{2 g}$ states to get the tight-binding model parameters for $\mathrm{SrTiO}_{3}$. We adopted maximally localized Wannier functions, following the prescription by Marzari et al. [37] and using the WIEN2WANNIER program [38], as shown in Fig. 5(b). With those hopping parameters, we construct a tight-binding lattice that on the $x / y$ plane keeps the infinite periodicity of the crystal, while along the $z$ direction we build a thick, real-space slab of up to 200 layers.

The lattice sites actually correspond to the $\mathrm{Ti}$ positions, while the effects of the oxygen orbitals are implicitly taken into account in the computation of the hopping integrals between the Wannier functions. We did not do any mass renormalization on the hopping parameters.

\section{APPENDIX B: DFT MODEL FOR TiO $\mathrm{T}_{2}$ ANATASE}

DFT calculations for $\mathrm{TiO}_{2}$ anatase were performed following the same procedure as for $\mathrm{SrTiO}_{3}$. The obtained bands are shown in Fig. 6. Note that anatase has two Ti atoms in the unit cell. Therefore, our Wannier basis set has six orbitals. We do consider, in this case, a mass renormalization factor of 0.6 , but its effect on the reported Fermi surfaces is rather minor.

\section{APPENDIX C: EFFECT OF THE FINITE PHOTOELECTRON ESCAPE LENGTH}

To quantify the effect of the finite inelastic mean free path of photoelectrons [Eq. (1)] compared to the case where such a damping is neglected, we show in Fig. 7 the effect of different photoelectron escape lengths $\lambda=5,10$, and $50 \AA$ on the simulation of the out-of-plane Fermi surface for the case of a 2DEG confined by a rectangular potential at the surface

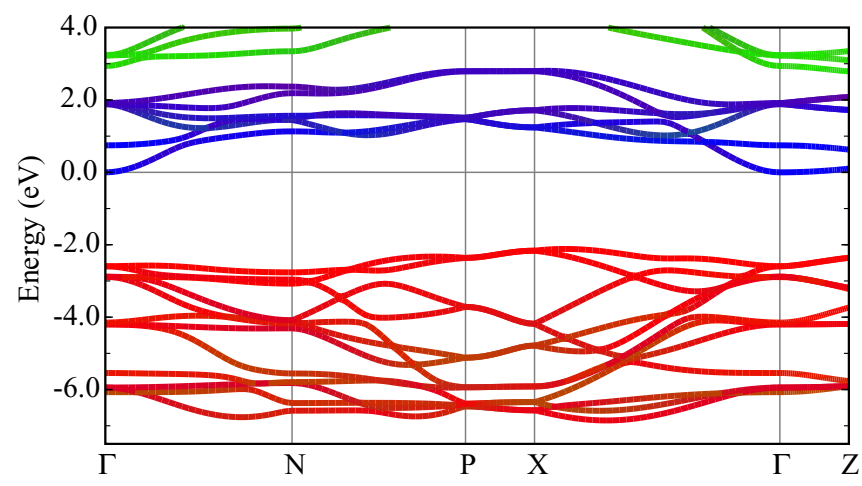

FIG. 6. DFT band structures for anatase. Red bands below $-2 \mathrm{eV}$ : oxygen orbitals; blue bands between the Fermi level and $\approx 3 \mathrm{eV}: t_{2 g}$ orbitals; green bands above $3 \mathrm{eV}: e_{g}$ orbitals. 

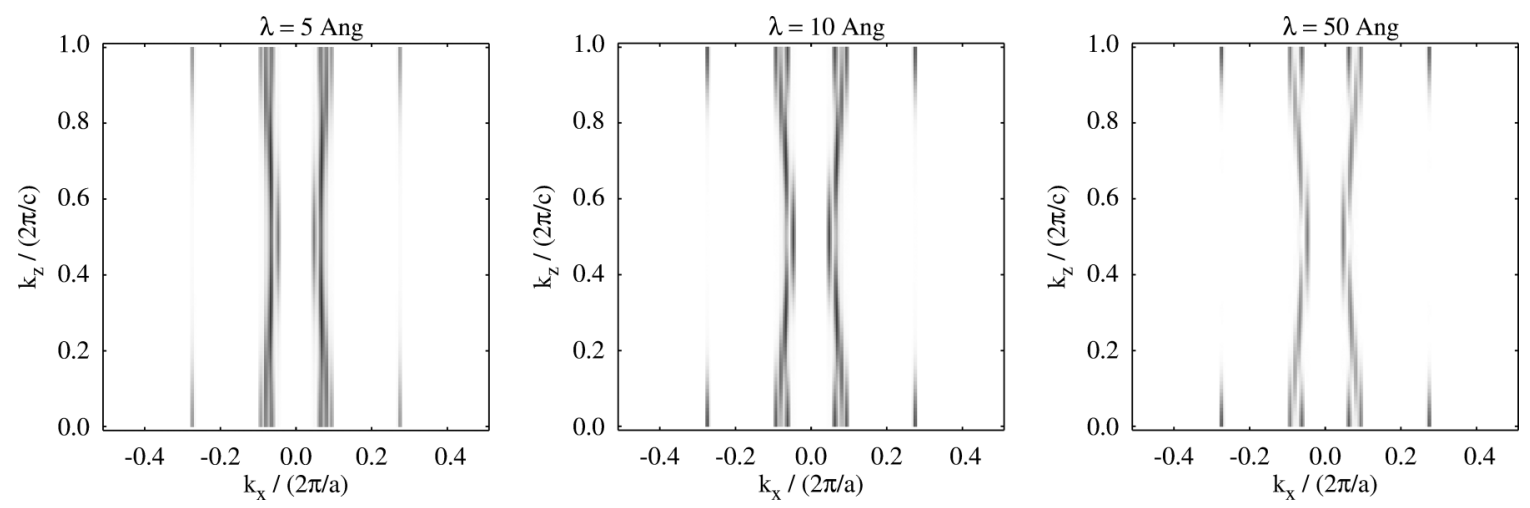

FIG. 7. Effect of the damping parameter $\lambda$ [Eq. (1)] on the plot of Fig. 1(c) for (from left to right) $\lambda=5 \AA, 10 \AA$, and $50 \AA$.

of $\mathrm{SrTiO}_{3}$ for a doping corresponding to Fig. 1(c). As seen by a comparison of those two figures, while the damping accentuates the $k_{z}$ modulation of the intensity, the effect caused by the interference of the electron wave function in the potential well remains the essential feature giving rise to the apparent dispersion of the out-of-plane Fermi surface.
[1] J. G. Bednorz and K. A. Müller, Possible high $\mathrm{T}_{C}$ superconductivity in the Ba-La-Cu-O system, Z. Phys. B 64, 189 (1986).

[2] E. Dagotto, Brief Introduction to Giant Magnetoresistance (GMR). Nanoscale Phase Separation and Colossal Magnetoresistance: The Physics of Manganites and Related Compounds (Springer Science \& Business Media, Berlin, Heidelberg, New York, 2003), pp. 395-396.

[3] M. Imada, Y. Tokura, and A. Fujimori, Metal-insulator transitions, Rev. Mod. Phys. 70, 1039 (1998).

[4] M. Rozenberg, Resistive Switching, http://www.scholarpedia. org/article/Resistive_switching.

[5] A. P. Ramirez, Oxide electronics emerge, Science 315, 1377 (2007).

[6] C. Cen, S. Thiel, J. Mannhart, and J. Levy, Oxide nanoelectronics on demand, Science 323, 1026 (2009).

[7] J. del Valle, J. G. Ramirez, M. J. Rozenberg, and I. K. Schuller, Challenges in materials and devices for resistive-switchingbased neuromorphic computing, J. Appl. Phys. 124, 211101 (2018).

[8] A. Ohtomo and H. Y. Hwang, A high-mobility electron gas at the $\mathrm{LaAlO}_{3} / \mathrm{SrTiO}_{3}$ heterointerface, Nature (London) 427, 423 (2004).

[9] A. F. Santander-Syro, O. Copie, T. Kondo, F. Fortuna, S. Pailhès, R. Weht, X. G. Qiu, F. Bertran, A. Nicolaou, A. Taleb-Ibrahimi, P. Le Fèvre, G. Herranz, M. Bibes, N. Reyren, Y. Apertet, P. Lecoeur, A. Barthélémy, and M. J. Rozenberg, Two-dimensional electron gas with universal subbands at the surface of $\mathrm{SrTiO}_{3}$, Nature (London) 469, 189 (2011).

[10] W. Meevasana, P. D. C. King, R. H. He, S.-K. Mo, M. Hashimoto, A. Tamai, P. Songsiriritthigul, F. Baumberger, and Z.-X. Shen, Creation and control of a two-dimensional electron liquid at the bare $\mathrm{SrTiO}_{3}$ surface, Nat. Mater. 10, 114 (2011).

[11] S. Moser, L. Moreschini, J. Jaćimović, O. S. Barišić, H. Berger, A. Magrez, Y. J. Chang, K. S. Kim, A. Bostwick, E. Rotenberg, L. Forró, and M. Grioni, Tunable Polaronic Conduction in Anatase $\mathrm{TiO}_{2}$, Phys. Rev. Lett. 110, 196403 (2013).
[12] T. C. Rödel, F. Fortuna, F. Bertran, M. Gabay, M. J. Rozenberg, A. F. Santander-Syro, and P. Le Fèvre, Engineering twodimensional electron gases at the (001) and (101) surfaces of $\mathrm{TiO}_{2}$ anatase using light, Phys. Rev. B 92, 041106(R) (2015).

[13] T. C. Rödel, F. Fortuna, S. Sengupta, E. Frantzeskakis, P. Le Fèvre, F. Bertran, B. Mercey, S. Matzen, G. Agnus, T. Maroutian, P. Lecoeur, and A. F. Santander-Syro, Universal fabrication of 2D electron systems in functional oxides, Adv. Mater. 28, 1976 (2016).

[14] T. C. Rödel, M. Vivek, F. Fortuna, P. Le Fèvre, F. Bertran, R. Weht, J. Goniakowski, M. Gabay, and A. F. Santander-Syro, Two-dimensional electron systems in $A_{\mathrm{TiO}}$ perovskites $(A=$ $\mathrm{Ca}, \mathrm{Ba}, \mathrm{Sr})$ : Control of orbital hybridization and energy order, Phys. Rev. B 96, 041121(R) (2017).

[15] A. F. Santander-Syro, C. Bareille, F. Fortuna, O. Copie, M. Gabay, F. Bertran, A. Taleb-Ibrahimi, P. Le Fèvre, G. Herranz, N. Reyren, M. Bibes, A. Barthélémy, P. Lecoeur, J. Guevara, and M. J. Rozenberg, Orbital symmetry reconstruction and strong mass renormalization in the two-dimensional electron gas at the surface of $\mathrm{KTaO}_{3}$, Phys. Rev. B 86, 121107(R) (2012).

[16] Z. Wang, Z. Zhong, X. Hao, S. Gerhold, B. Stöger, M. Schmid, J. Sánchez-Barriga, A. Varykhalov, C. Franchini, K. Held, and U. Diebold, Anisotropic two-dimensional electron gas at $\mathrm{SrTiO}_{3}$ (110), Proc. Natl. Acad. Sci. USA 111, 3933 (2014).

[17] T. C. Rödel, C. Bareille, F. Fortuna, C. Baumier, F. Bertran, P. Le Fèvre, M. Gabay, O. Hijano Cubelos, M. J. Rozenberg, T. Maroutian, P. Lecoeur, and A. F. Santander-Syro, Orientational Tuning of the Fermi Sea of Confined Electrons at the $\mathrm{SrTiO}_{3}$ (110) and (111) Surfaces, Phys. Rev. Appl. 1, 051002 (2014).

[18] S. Backes, T. C. Rödel, F. Fortuna, E. Frantzeskakis, P. Le Fèvre, F. Bertran, M. Kobayashi, R. Yukawa, T. Mitsuhashi, M. Kitamura, K. Horiba, H. Kumigashira, R. Saint-Martin, A. Fouchet, B. Berini, Y. Dumont, A. J. Kim, F. Lechermann, H. O. Jeschke, M. J. Rozenberg, R. Valentí, and A. F. Santander-Syro, Hubbard band versus oxygen vacancy states in the correlated electron metal $\mathrm{SrVO}_{3}$, Phys. Rev. B 94, 241110(R) (2016). 
[19] E. Frantzeskakis, T. C. Rödel, F. Fortuna, and A. F. SantanderSyro, 2D surprises at the surface of 3D materials: Confined electron systems in transition metal oxides, J. Electron Spectrosc. Relat. Phenom. 219, 16 (2017).

[20] P. Lömker, T. C. Rödel, T. Gerber, F. Fortuna, E. Frantzeskakis, P. Le Fèvre, F. Bertran, M. Müller, and A. F. Santander-Syro, Two-dimensional electron system at the magnetically tunable $\mathrm{EuO} / \mathrm{SrTiO}_{3}$ interface, Phys. Rev. Mater. 1, 062001(R) (2017).

[21] T. C. Rödel, J. Dai, F. Fortuna, E. Frantzeskakis, P. Le Fèvre, F. Bertran, M. Kobayashi, R. Yukawa, T. Mitsuhashi, M. Kitamura, K. Horiba, H. Kumigashira, and A. F. SantanderSyro, High-density two-dimensional electron system induced by oxygen vacancies in $\mathrm{ZnO}$, Phys. Rev. Mater. 2, 051601(R) (2018).

[22] M. Altmeyer, H. O. Jeschke, O. Hijano-Cubelos, C. Martins, F. Lechermann, K. Koepernik, A. F. Santander-Syro, M. J. Rozenberg, R. Valentí, and M. Gabay, Magnetism, SpinTexture, and In-Gap States: Atomic Specialization at the Surface of Oxygen-Deficient $\mathrm{SrTiO}_{3}$, Phys. Rev. Lett. 116, 157203 (2016).

[23] C. G. Van de Walle, Hydrogen as a Cause of Doping in Zinc Oxide. Phys. Rev. Lett. 85, 1012 (2000).

[24] S. Sengupta, E. Tisserond, F. Linez, M. Monteverde, A. Murani, T. C. Rödel, P. Lecoeur, T. Maroutian, C. Marrache-Kikuchi, A. F. Santander-Syro, and F. Fortuna, Gate-tunable superconductivity at $\mathrm{SrTiO}_{3}$ surface realized by $\mathrm{Al}$ layer evaporation, J. App. Phys. 124, 213902 (2018).

[25] N. C. Plumb, M. Salluzzo, E. Razzoli, M. Månsson, M. Falub, J. Krempasky, C. E. Matt, J. Chang, M. Schulte, J. Braun, H. Ebert, J. Minár, B. Delley, K.-J. Zhou, T. Schmitt, M. Shi, J. Mesot, L. Patthey, and M. Radović, Mixed Dimensionality of Confined Conducting Electrons in the Surface Region of $\mathrm{SrTiO}_{3}$, Phys. Rev. Lett. 113, 086801 (2014).

[26] S. Moser, An experimentalist's guide to the matrix element in angle resolved photoemission, J. Electron Spectrosc. Relat. Phenom. 214, 29 (2017).

[27] S. Moser, V. Jovic, R. Koch, L. Moreschini, J.-S. Oh, C. Jozwiak, A. Bostwick, and E. Rotenberg, How to extract the surface potential profile from the ARPES signature of a 2DEG, J. Electron Spectrosc. Relat. Phenom. 225, 16 (2018).
[28] L. F. Mattheiss, Effect of the $110 \mathrm{~K}$ transition on the $\mathrm{SrTiO}_{3}$ conduction bands, Phys. Rev. B 6, 4740 (1972).

[29] P. D. C. King, S. McKeown Walker, A. Tamai, A. de la Torre, T. Eknapakul, P. Buaphet, S.-K. Mo, W. Meevasana, M. S. Bahramy, and F. Baumberger, Quasiparticle dynamics and spinorbital texture of the $\mathrm{SrTiO}_{3}$ two-dimensional electron gas, Nat. Commun. 5, 3414 (2014).

[30] M. Landmann, E. Rauls, and W. G. Schmidt, The electronic structure and optical response of rutile, anatase and brookite $\mathrm{TiO}_{2}$, J. Phys.: Condens. Matter 24, 195503 (2012).

[31] S. Hüfner, Photoelectron Spectroscopy. Principles and Applications, 3rd ed. (Springer-Verlag, Berlin, 2003).

[32] J. W. Gadzuk, Surface molecules and chemisorption. II. Photoemission angular distributions, Phys. Rev. B 10, 5030 (1974).

[33] A. Mugarza, J. E. Ortega, F. J. Himpsel, and F. G. García de Abajo, Measurement of electron wave functions and confining potentials via photoemission, Phys. Rev. B 67, 081404(R) (2003).

[34] P. Puschnig, S. Berkebile, A. J. Fleming, G. Koller, K. Emtsev, T. Seyller, J. D. Riley, C. Ambrosch-Draxl, F. P. Netzer, and M. G. Ramsey, Reconstruction of molecular orbital densities from photoemission data, Science 326, 702 (2009).

[35] P. Pusching and D. Lüftner, Simulation of angle-resolved photoemission spectra by approximating the final state by a plane wave: From graphene to polycyclic aromatic hydrocarbon molecules, J. Electron Spectrosc. Relat. Phenom. 200, 193 (2015).

[36] P. Blaha, K. Schwarz, G. K. H. Madsen, D. Kvasnicka, J. Luitz, R. Laskowski, F. Tran, and L. D. Marks, WIEN2k, An Augmented Plane Wave + Local Orbitals Program for Calculating Crystal Properties (Karlheinz Schwarz, Techn. Universität Wien, Austria, 2018).

[37] A. A. Mostofi, J. R. Yates, G. Pizzi, Y. S. Lee, I. Souza, D. Vanderbilt, and N. Marzari, An updated version of WANNIER90: A tool for obtaining maximally-localised Wannier functions, Comput. Phys. Commun. 185, 2309 (2014).

[38] J. Kunes, R. Arita, P. Wissgott, A. Toschi, H. Ikeda, and K. Held, wIEN2WANNIER: From linearized augmented plane waves to maximally localized Wannier functions, Comput. Phys. Commun. 181, 1888 (2010). 